\title{
Board of Directors and Officers of the Li Fang-Kuei Society for Chinese Linguistics \\ 紀念李方桂先生中國語言學研究學會董事及執行委員名單
}

Honorary President for Life 終身榮譽會長: Pang-Hsin Ting 丁邦新

(Agassiz Professor Emeritus, University of California, Berkeley; Professor Emeritus, Hong Kong University of Science and Technology 加州大學柏克萊校區榮休 Agassiz 講座教授、香港科技大學榮休教授)

2003-2008 Founding Board of Directors 創會董事名單:

Hung-Nin Samuel Cheung 張洪年 (Professor Emeritus, University of California, Berkeley; Professor, Hong Kong University of Science and Technology 加州大學柏克萊校區榮休教授、香港科技大學教授); South Coblin 柯蔚南 (Professor, Iowa University 愛我華大學教授); C.-T. James Huang 黃正德 (Professor, Harvard University 哈佛大學教授); Tsu-Lin Mei 梅祖麟 (Hu Shih Professor Emeritus, Cornell University 康 奈爾大學榮休胡適講座教授); Ken-ichi Takashima 高嶋謙一 (Professor, University of British Columbia 英屬哥倫比亞大學教授); Pang-Hsin Ting 丁邦新 (Agassiz Professor Emeritus, University of California, Berkeley; Professor, Hong Kong University of Science and Technology 加州大學柏克萊校區榮休 Agassiz 講座教授、香港科技大學教授); Anne Yue-Hashimoto 余䨠芹 (Professor, University of Washington 華 盛頓大學教授)

2003-2008 Founding Officers 創會執行委員名單:

President 會長: Pang-Hsin Ting 丁邦新

Executive Secretary cum Treasurer 秘書長兼司庫: Anne Yue-Hashimoto 余靄芹

Deputy Secretary 副秘書: Zhongwei Shen 沈鍾偉 (Assistant Professor, University of Massachusetts Amherst)

2008-2011 Board of Directors 董事名單:

Wolfgang Behr 畢鶚 (Associate Professor, Universität Zürich 蘇黎世大學副教授) (2009-2011); Hung-Nin Samuel Cheung 張洪年 (Professor Emeritus, University of California, Berkeley; Chair Professor, Chinese University of Hong Kong, 加州大學柏克萊校區榮休教授、香港中文大學講座教授); South Coblin 柯 蔚南 (Professor, Iowa University 愛我華大學教授); Zev Handel 韓哲夫 (Associate Professor, University of Washington 華盛頓大學副教授); Dah-An Ho 何大安 (Research Fellow and Director Emeritus, Institute of Linguistics, Academia Sinica 中央研究院語言學研究所研究員兼榮休所長); C.-T. James Huang 黃正德 (Professor, Harvard University 哈佛大學教授); Tsu-Lin Mei 梅祖麟 (Hu Shih Professor Emeritus, Cornell University 康奈爾大學榮休胡適講座教授) (2008-2009); Zhongwei Shen 沈鍾偉 (Associate Professor, University of Massachusetts Amherst 馬薩諸塞大學安默斯特校區副教授); Ken-ichi Takashima 高嶋謙一 (Professor Emeritus, University of British Columbia 英屬哥倫比亞大學榮休教授); Anne YueHashimoto 余雹芹 (Professor, University of Washington 華盛頓大學教授) 
2008-2011 Officers 執行委員名單:

President 會長: South Coblin 柯蔚南

Executive Secretary 秘書長: Hung-Nin Samuel Cheung 張洪年

Treasurer 司庫: Anne Yue-Hashimoto 余霭芹

Deputy Secretary 副秘書: Zhongwei Shen 沈鍾偉

2011-2014 Board of Directors 董事名單:

Wolfgang Behr 畢鶚 (Professor and Traditional China Chair, Institute of Asian and Oriental Studies, Universität Zürich 蘇黎世大學東亞傳統中國講座教授); Hung-Nin Samuel Cheung 張洪年 (Professor Emeritus, University of California, Berkeley; Chair Professor Emeritus, Chinese University of Hong Kong 加州大 學柏克萊校區榮休教授、香港中文大學榮休講座教授); South Coblin 柯蔚南 (Professor Emeritus, Iowa University 愛我華大學榮休教授); Zev Handel 韓哲夫 (Associate Professor, University of Washington 華盛頓大學副教授); Dah-An Ho 何大安 (Research Fellow and Director Emeritus, Institute of Linguistics, Academia Sinica 中央研究院語言學研究所研究員兼榮休所長); C.-T. James Huang 黃正德 (Professor, Harvard University 哈佛大學教授); Zhongwei Shen 沈鍾偉 (Professor, University of Massachusetts Amherst 馬薩諸塞大學安默斯特校區教授); Ken-ichi Takashima 高嶋謙一 (Professor Emeritus, University of British Columbia; Visiting Professor, Anhui University 英屬哥倫比亞大學榮休教授、安徽大學客 座教授); Anne Yue-Hashimoto 余靄芹 (Professor, University of Washington 華盛頓大學教授)

2011-2014 Officers 執行委員名單:

President 會長: South Coblin 柯蔚南

Executive Secretary 秘書長: Zhongwei Shen 沈鍾偉

Treasurer 司庫: Anne Yue-Hashimoto 余靄芹

Deputy Secretary 副秘書: Zev Handel 韓哲夫

\section{4-2017 Board of Directors 董事名單:}

Wolfgang Behr 畢鶚 (Professor and Traditional China Chair, Institute of Asian and Oriental Studies, Universität Zürich 蘇黎世大學東亞傳統中國講座教授); Zhongmin Chen 陳忠敏 (Professor, Fudan University 復旦大學教授) (2015-2018); Zev Handel 韓哲夫 (Associate Professor, University of Washington 華盛頓大學副教授); Dah-An Ho 何大安 (Research Fellow and Director Emeritus, Institute of Linguistics, Academia Sinica 中央研究院語言學研究所研究員兼榮休所長); C.-T. James Huang 黃正德 (Professor, Harvard University 哈佛大學教授); Zhongwei Shen 沈鍾偉 (Professor, University of Massachusetts Amherst 馬薩諸塞大學安默斯特校區教授); Ken-ichi Takashima 高嶋謙一 (Professor Emeritus, University of British Columbia; Distinguished Visiting Professor, Anhui University 英屬哥倫比亞大學榮休教 授、安徽大學特聘教授); Rui-wen Wu 吳瑞文 (Associate Research Fellow, Institute of Linguistics, Academia Sinica 中央研究院語言學研究所副研究員) (2015-2018); Anne Yue-Hashimoto 余霰芹 (Professor, University of Washington 華盛頓大學教授)

2014-2017 Officers 執行委員名單:

President 會長: Anne Yue-Hashimoto 余靄芹

Executive Secretary 秘書長: Zhongwei Shen 沈鍾偉

Treasurer 司庫: Zev Handel 韓哲夫 $\left(2015^{-2017}\right)$

Deputy Secretary 副秘書: Zev Handel 韓哲夫 
2017-2020 Board of Directors 董事名單:

Wolfgang Behr 畢鶚 (Professor and Traditional China Chair, Institute of Asian and Oriental Studies, Universität Zürich 蘇黎世大學東亞傳統中國講座教授); Zhongmin Chen 陳忠敏 (Professor, Fudan University 復旦大學教授); Zev Handel 韓哲夫 (Associate Professor, University of Washington 華盛頓大學 副教授); C.-T. James Huang 黃正德 (Professor, Harvard University 哈佛大學教授); Zhongwei Shen 沈鍾 偉 (Professor, University of Massachusetts Amherst 馬薩諸塞大學安默斯特校區教授); Jackson T.-S. Sun (Distinguished Research Fellow, Institute of Linguistics, Academia Sinica 中央研究院語言學研究所特聘 研究員) (2018-2023); Ken-ichi Takashima 高嶋謙一 (Professor Emeritus, University of British Columbia; Distinguished Professor, Anhui University 英屬哥倫比亞大學榮休教授、安徽大學特聘教授) (20172018); Rui-wen Wu 吳瑞文 (Research Fellow, Institute of Linguistics, Academia Sinica 中央研究院語言 學研究所研究員); Hsiu-fang Yang 楊秀芳 (Professor Emerita, National Taiwan University 國立台灣大學 榮休教授); Anne Yue-Hashimoto 余䨠芹 (Professor Emerita 2018, University of Washington 華盛頓大學 榮休教授)

2017-2020 Officers 執行委員名單:

President 會長: Anne Yue-Hashimoto 余靄芹

Executive Secretary 秘書長: Hsiu-fang Yang 楊秀芳

Bilingual Secretary 雙語秘書: Bit-Chee Kwok 郭必之 (Associate Professor, The Chinese University of Hong Kong 香港中文大學副教授) (2018-2023)

Treasurer 司庫: Zev Handel 韓哲夫

Deputy Secretary 副秘書: Zev Handel 韓哲夫 (2017-2018); Min-hua Chiang 江敏華 (Research Fellow, Institute of Linguistics, Academia Sinica 中央研究院語言學研究所研究員) (2018-2023)

Assistant to the President 會長助理: Rui-wen Wu 吳瑞文 (2018-2023)

Officer of Web Site Management 網站管理員: Andy Chin 錢志安 (Associate Professor, The Education University of Hong Kong 香港教育大學副教授)

Officer/Member of the Field Work Award Committee: Weera Ostapirat (Associate Professor of Linguistics) (2018-2023) 